\title{
Physicochemical and Biological Investigation of Different Structures of Carbon Coatings Deposited onto Polyurethane
}

\author{
Witold Kaczorowski* Damian Batory ${ }^{1}$, Witold Jakubowski ${ }^{1}$, Witold Szymanski ${ }^{1}$, Piotr \\ Komorowski $^{2}$, Bogdan Walkowiak ${ }^{1}$, Marek Sanak ${ }^{3}$ and Piotr Niedzielski ${ }^{1}$ \\ ${ }^{1}$ Lodz University of Technology; Institute of Materials Science and Engineering, Stefanowskiego St. - Poland. \\ ${ }^{2}$ BioNanoPark Laboratories of Lodz Regional Park of Science and Technology; Lodz - Poland. ${ }^{3}$ Jagiellonian \\ University Medical College; Krakow - Poland
}

\begin{abstract}
The aim of this study was to examine the thrombogenic properties of polyurethane that was surface modified with carbon coatings. Physicochemical properties of manufactured coatings were investigated using transmission electron microscopy (TEM), atomic force microscopy (AFM), X-ray Photoelectron Spectroscopy (XPS), Raman spectroscopy and contact angle measurement methods. Samples were examined by the Impact- $R$ method evaluating the level of platelets activation and adhesion of particular blood cell elements. The analysis of antimicrobial resistance against E. coli colonization and viability of endothelial cells showed that polyurethane modified with use of carbon layers constituted an interesting solution for biomedical application.
\end{abstract}

Key words: Diamond-like carbon, Atomic force microscopy (AFM), X-ray photoelectron spectroscopy XPS, Raman spectroscopy, biological properties

\section{INTRODUCTION}

Polymeric biomaterials increasingly displace standard materials used in the medical field such as steels and other metal alloys. They are indispensable in the manufacturing of artificial veins, eyeballs, equipment and other medical instruments and implants. However, among the medical fields, where polymer materials can be used, the most demanding application seems to be the construction of an artificial heart. Here, in addition to the good mechanical properties, the highest hemo-, thrombo- and biocompatibility are absolutely required.

It is know that in case of polymeric implants having contact with blood, in order to avoid the risk of thrombus formation, it is necessary to modify their surface (Courtney et al. 1994; Schroeder et al.
2000). For this purpose, different bioactive substances are used (heparin, albumin and other) (Junkar et al. 2009; Bazaka et al. 2011). There are several reports on the use of different types of thin coatings produced with the use of plasma techniques (Ohgoe et al. 2004; Jones et al. 2006; Desmet et al. 2009; Bozukova et al. 2010). The plasma treatment leads the synthesis of thin coatings and allows the functionalization of the modified surface. However, the practical application of plasma modification of polymers is not easy due to its negative effect on both the aging processes and the surface development (Junkar et al. 2009).

Diamond-like coating (DLC) is a metastable form of amorphous carbon containing both $\mathrm{sp}^{2}$ (graphite like) bonds and $\mathrm{sp}^{3}$ (diamond-like) bonds. The hydrogenated $(\mathrm{a}-\mathrm{C}: \mathrm{H})$ and non-hydrogenated $(\mathrm{a}-\mathrm{C})$

*Author for correspondence: witold.kaczorowski@p.lodz.pl 
as well as tetrahedrally coordinated amorphous carbons (ta-C) are referred to as DLC (Robertson, 2002), whereas the $\mathrm{sp}^{3} / \mathrm{sp}^{2}$ bonded carbon ratio are important factors influencing the biological response of DLC surfaces (Ma et al. 2007). These coatings have become quite popular in biomedical applications, particularly in cardiovascular, orthopaedic and ophthalmic applications (Narayan 2005; Mitura et al. 2006; Kleinen et al. 2008; Fedel et al. 2009; Lackner et al. 2009; Asakawa et al. 2011; Batory et al. 2012; Batory et al. 2014). They are also used in the modification of the surface of polyurethane presenting good flexibility, still maintaining high level of adhesion (Ohgoe et al. 2004; Lackner et al. 2009). These films demonstrate very high hemocompatibility and lack of cytotoxity (Matsumoto et al. 2008; Lackner et al. 2012). They decrease platelet activation and reduce thrombus formation on a cardiovascular device surface. Their extremely low coefficient of friction may increase the rate of the blood flow and improve other hemodynamic properties (Hauert 2004; Hauert and Muller 2003; Narayan 2005). As shown in the clinical practice, their very good resistance against bacterial colonisation makes them a perfect material for the applications preventing biofilm formation (Laube et al. 2007).

One of the most commonly used methods of the synthesis of carbon coatings on polyurethane substrates is RF PACVD method (Nakahigashi et al.2004; Ohgoe et al. 2004; Jones et al. 2006; Matsumoto et al. 2008; Martinez-Martinez et al. 2014). Despite its popularity, it has several weaknesses. One of them is relatively high level of the residual stress of synthesized coatings. The other is low density of high energy ions, which in consequence, due to application of high RF powers, may lead to noticeable degradation of the modified surface. In view of the above, this study aimed to develop two different methods for the preparation of carbon coatings on polymer substrates, which could reduce the described drawbacks. The first one, RF PACVD/MS (Radio Frequency Plasma Assisted Chemical Vapour Deposition / Magnetron Sputtering) allows the production of carbon coatings with gradient $\mathrm{Ti}^{-} \mathrm{Ti}_{\mathrm{x}} \mathrm{C}_{\mathrm{y}}$-DLC interlayer, which greatly reduces the stress and improves the adhesion of the final carbon coatings on the top. The second one, MW/RF PA CVD (Microwave / Radio Frequency Plasma Assisted Chemical Vapor Deposition) allows modification of polymer substrates in the dual frequency plasma, where the MW plasma determines the high density of ions, whereas RF plasma controls their energy. Therefore, it is possible to synthesize carbon coatings in the lower energy regimes compared to pure RF plasma. Other advantages of this technology have been described by Kaczorowski et al. (2008).

\section{MATERIAL AND METHODS}

Samples were flat discs 40 and $8 \mathrm{~mm}$ in diameter and 2.5 and $2 \mathrm{~mm}$ length, made of polyurethane (ChronoFlex). Before the synthesis, the specimens were cleaned ultrasonically in isopropyl alcohol bath for 10 minutes, then dried using the compressed air and placed on r.f. (radio frequency) electrodes of plasmochemical reactors. Processes of modification were conducted using two plasma modification technologies as described elsewhere (Batory et al. 2008; Kaczorowski and Niedzielski 2008; Batory et al. 2013; Kaczorowski et al. 2014). Dual-frequency technology (MW/RF PACVD method marked as MW/RF) was used in order to conduct the modification processes using pure methane. Carbon coatings were synthesized using $\mathrm{CH}_{4}$ atmosphere under the pressure of $100 \mathrm{~Pa}$ in 5 min. Negative r.f. bias was equal to $250 \mathrm{~V}$, whereas the microwave power, introduced into the chamber via quartz tube, was adjusted to $900 \mathrm{~W}$. The hybrid technology of DC pulsed magnetron sputtering supported by the radio frequency discharge (RF PACVD/MS method marked as RF/MS) was used in order to conduct the modification in two stage process. The process of synthesis was preceded by plasma etching of the polymer substrate. The etching parameters were optimised to guarantee the good adhesion of carbon coatings to PU substrates (Kaczorowski et al. 2009). Combination of CVD (Chemical Vapour Deposition) and PVD (Physical Vapour Deposition) allows application of an additional $\mathrm{Ti}-\mathrm{Ti}_{\mathrm{x}} \mathrm{C}_{\mathrm{y}}$ gradient interlayer as stress absorbing buffer giving higher flexibility of the coating (Lackner et al. 2009). In order to improve the adhesion of $\mathrm{Ti}$ interlayer to PU substrate, the etching process was conducted for $10 \mathrm{~min}$ in $\mathrm{O}_{2} / \mathrm{Ar}$ (50/50 ratio) atmosphere under the pressure of $1 \mathrm{~Pa}$ and negative r.f. bias of $540 \mathrm{~V}$. After the etching process, $\mathrm{Ti}-\mathrm{Ti}_{\mathrm{x}} \mathrm{C}_{\mathrm{y}}$ gradient interlayer was synthesized using the magnetron sputtering process of Ti cathode in $\mathrm{Ar} / \mathrm{CH}_{4}$ atmosphere, conducted in the radio frequency electric field under negative r.f. bias of $300 \mathrm{~V}$. Ar/ $\mathrm{CH}_{4}$ composition ratio as well as the sputtering parameters were matched to obtain 
the gradient of chemical composition form pure $\mathrm{Ti}$ to $\mathrm{Ti}_{\mathrm{x}} \mathrm{C}_{\mathrm{y}}-$ DLC composite. Final carbon coating was synthesized by the means of RF PACVD method from methane atmosphere under pressure of $20 \mathrm{~Pa}$, negative r.f. bias of $500 \mathrm{~V}$ for time of 5 $\min$.

Structure and thickness of the manufactured coatings were examined using the transmission electron microscope Tecnai G2 F20 (200kV), equipped with high resolution CCD camera (GatanUltraScan). Specimens for the examinations were prepared using the focused ion beam (FIB) system equipped with $\mathrm{Ga}+$ ions source. Surface morphology and topography were measured using AFM Multimode microscope equipped with Nanoscope V controller (Bruker Corporation, USA) working in tapping mode. Image acquisition was performed with the use of Nanoscope 7.3 software and further image processing was done using MountainsMap 5 (Digital Surf, France) software. For each sample, the areas of $100 \times 100$ $\mu \mathrm{m}$ and $1 \mathrm{x} 1 \mu \mathrm{m}$ were scanned and roughness parameters were determined (average values taken from 512 surface profiles). Surface free energy (SFE) was determined according to the OwensWendt method (Rudawska and Jacniacka, 2009; Myllymaa et al. 2013;). Contact angles were measured with KRUSS Contact Angle Measuring Instrument, using the sessile drop method. The investigation was made for deionised water and diiodomethane (Sigma Aldrich, USA). Each time a standard volume of $0.8 \mu \mathrm{L}$ was applied on the sample surface. The measurement was repeated three times and the results were averaged. Surface functionalization was determined by $\mathrm{X}$-ray photoelectron spectroscopy R3000 (VG Scienta, Sweden) system equipped with monochromatic Al $(\mathrm{K} \alpha 1,2=1486.6 \mathrm{eV}) \mathrm{X}$-ray source. The calibrations of peak position were done in accordance to $\mathrm{C} 1 \mathrm{~s}$ peak energy established at $284.6 \mathrm{eV}$ (Tai et al. 2009; Wang et al. 2009). The $\mathrm{C} 1 \mathrm{~s}$ core level spectra were obtained at pass energy of $25 \mathrm{eV}$. Experimental spectra were analysed using PEAKFIT software. Chemical structure of the synthesized coatings was determined using inVia confocal micro-Raman spectrometer (Renishaw), working with $532 \mathrm{~nm}$ wavelength and power of $2.5 \mathrm{~mW}$.

The carbon coatings were tested under conditions modelling dynamic contact with whole human blood under the shear stress (the Impact-R method). In brief, donor's venous blood was collected on sodium citrate $30 \mathrm{~min}$ before a test. Tested surfaces were disks made of the polyurethane or polyurethane with the carbon coatings. Disks were fitted into experimental wells, overlayed with anticoagulated blood, and subsequently shear stress was applied during $300 \mathrm{~s}$ using a disposable conical rotor revolving $720 \mathrm{rpm}$. This corresponded to a shear rate of 1800/s, typical for arterial blood flow. Following the shear stress, blood was collected from the wells. Expression of platelet activation markers and platelet aggregates was measured by flow cytometry using fluorescently labelled monoclonal antibodies against selectin-P activation marker (CD62P) and platelet-specific integrin GPIIIa (CD61) gating platelets. Total platelets count was measured using automated cell counter (Sysmex-K1000, Sysmex, USA). The degree of fragmentation of activated platelets was measured as plateled-derived microparticles concentration in the plasma using an immuno-enzymatic assay. Thus, cell membrane phospholipids of the fragmented platelet were quantified (Zymuphen MP-activity ELISA kit; Hyphen Biomed, Eragny, France), following Sanak et al. (2010). Results comparing polyurethane with the tested coatings were calculated following the subtraction of baseline parameters of the donor's blood.

All the tests were done in six replicates, an additional functional test of platelets activated using adenosine diphosphate $[5 \mu \mathrm{M}]$ was used as a positive control of platelet reactivity. Samples for the bacterial colonisation were placed each into a homemade bioreactor $(200 \mathrm{~mL})$ and were immersed in the medium containing $\mathrm{NaCl}(1 \%)$, bactopeptone $(1 \%)$ and yeast extract $(0.5 \%), \mathrm{pH} 7.0$. The medium was supplemented with a small number (approximately $2 \times 10^{3}$ ) of $E$. coli cells (strain DH5 $\alpha$ ). The cells were cultured at $37^{\circ} \mathrm{C}$ for $24 \mathrm{~h}$. Samples removed from the culture media were washed extensively with deionised water. Next, bacterial cells present on the sample surfaces were visualized using Olympus GX71 microscope (Olympus, Center Valley, USA), equipped with a CCD camera (DC 70). Control samples, made of polished AISI 3161 steel, were present and treated identically in each experiment. Samples were processed in two separate experiments, and up to six randomly selected separate areas were inspected for each sample (Jakubowski et al. 2004). For acquired microscopic images, Image $\mathbf{J}$ software was used to calculate the area of bacterial surface coverage. Endothelial cells (immortalized cell line EA.hy 926, ATCC, Manassas, USA) were cultured in tissue culture plastics (TPP, Trasadingen, Switzerland) using Dulbecso's modified Eagle's 
medium with high glucose concentration $(4.5 \mathrm{~g} / \mathrm{L})$, containing $10 \% \mathrm{FBS}$ and antibiotics, at $37^{\circ} \mathrm{C}$ in a humidified atmosphere containing $5 \% \mathrm{CO}_{2}$. The cells were cultured on the investigated surfaces for $48 \mathrm{~h}$. For the control, cells cultured in the standard conditions were used. Samples cytotoxicity was estimated by live/dead test using calcein-AM and ethidiumhomodimer

(Live/Dead Viability/Cytotoxicity Kit, Molecular Probes, Eugene, USA) and Olympus GX71 fluorescence microscope.

\section{RESULTS AND DISCUSSION}

\section{The structure of coatings}

Figure 1 presents the structures of PU substrates modified using the MW/RF technique and RF/MS. In the first type of structure of carbon coating (obtained using MW/RF plasma), the thickness of analyzed coatings was around $20-30 \mathrm{~nm}$ (Fig. 1A). The interface between the manufactured film and polyurethane substrate was barely visible due to both coating and substrate amorphous structure. Nevertheless, it appeared to be continuous and uniform.

Figures $1 \mathrm{~B}$ and $\mathrm{C}$ present the structure of gradient nano-composite carbon coating deposited using the RF PACVD/MS technique. Structure of the coating consisted of three distinguishable zones of the total thickness of about $180 \mathrm{~nm}$. The first one was $\mathrm{Ti}$ interlayer deposited directly on the substrate surface. The nanocomposite interlayer was visible where the nano-crystalline TixCy inclusions were randomly distributed in an amorphous matrix of diamond-like carbon (Fig. 1C). The external zone of the coating was amorphous carbon layer. Voids in the cross-section area of the nanocomposite interlayer were rather the result of the sample preparation procedure than the process of synthesis itself. The adhesion of both types of the coatings appeared to be satisfactory since no cracks or other discontinuities were observed neither in the coating, nor in the interface with the substrate, despite of simple bending tests performed before the thin foil preparation procedure. HRTEM examination results proved the continuous interface between both the coatings and modified substrates.
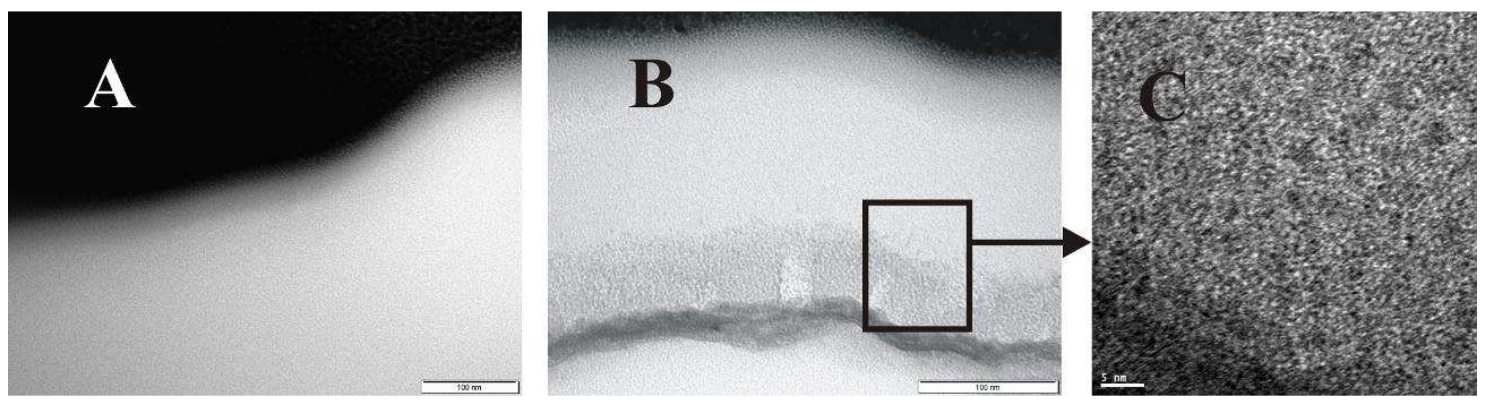

Figure 1 - TEM of PU substrate modified with carbon coatings (obtained using MW/RF plasma) (A) and gradient titanium carbon layer (obtained using RF/MS plasma) (B). High-resolution view of the $\mathrm{Ti}-\mathrm{Ti}_{\mathrm{x}} \mathrm{C}_{\mathrm{y}}-\mathrm{DLC}$ nanocomposite interlayer $(\mathrm{C})$.

\section{The Analysis of Surface Geometry Changes}

Figure 2 shows the AFM topography scans of examined samples. Table 1 shows the roughness parameters determined for the areas of $100 \times 100$ $\mu \mathrm{m}$. Surface of PU substrate (Fig. 2B) was characterized by the low values of Ra, RMS and Rmax roughness parameters equal to 12.9, 16.5 and $325 \mathrm{~nm}$, respectively. Both modification processes resulted in increased values of these parameters, amounting up to 146, 178 and $1459 \mathrm{~nm}$ for MW/RF method (Fig. 2D) and 186, 225 and $1709 \mathrm{~nm}$ for RF/MS method (Fig. 2F).
Table 1- Roughness parameters for bare substrate, substrate modified by MW/RF and RF/MS methods (determined for scanned areas of $100 \times 100 \mu \mathrm{m}$ ).

\begin{tabular}{cccc}
\hline Sample & Ra [nm] & RMS [nm] & Rmax [nm] \\
\hline PU & 12.9 & 16.5 & 325 \\
MW/RF & 146 & 178 & 1459 \\
RF/MS & 186 & 225 & 1709 \\
\hline
\end{tabular}

Obviously, modification of the polyurethane substrates by means of both synthesis techniques resulted in the higher surface development. Both coatings appeared to be quite uniform with the homogeneous topographical pattern, which was 
smoother for MW/RF and coarser for RF/MS technique.
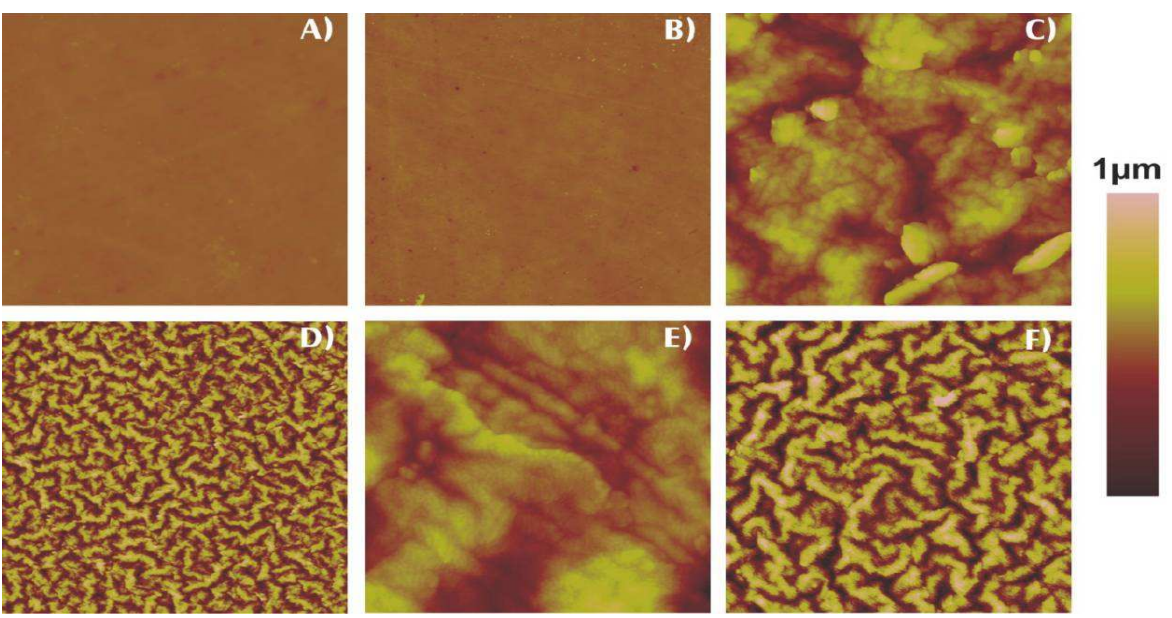

Figure 2 - AFM topography images: PU substrate (A, B), substrate modified with use of MW/RF method (C,D), substrate modified with use of RF/MS method (E,F). Scanned areas: 10 x 10 $\mu \mathrm{m}(\mathrm{A}, \mathrm{C}$ and $\mathrm{E})$ and 100x100 $\mu \mathrm{m}(\mathrm{B}, \mathrm{D}$ and $\mathrm{F})$.

\section{Material Characteristics}

The study of the chemical composition and chemical structure of modified and unmodified PU substrates was carried out based on XPS and Raman spectroscopy results. The basic chemical composition from XPS investigations of examined samples before and after the modification is presented in Table 2. As it was expected, after the synthesis of carbon-based coatings, the PU samples were characterized by increased concentration of carbon and decreased concentration of oxygen. The deconvoluted XPS C1s core level spectra for the modified carbon-based coatings are presented in Figure 3. Four Gaussian profiles with characteristic binding energies, associated with a certain chemical state of a film component, were fitted to each $\mathrm{C} 1 \mathrm{~s}$ peak. The carbon peaks at binding energies of $\sim 284.5 \mathrm{eV}$ and $\sim 285.3 \mathrm{eV}$ corresponded to $\mathrm{sp}^{2}$ and $\mathrm{sp}^{3}$ coordinated carbon bonding, respectively (Narayan 2005; Kaczorowski et al. 2014; Batory et al. 2015). The other two carbon peaks located at binding energies of $\sim 286.1 \mathrm{eV}$ and $\sim 288 \mathrm{eV}$ were associated with oxygen adsorbed on the coatings surface (Foong et al. 2011; Takabayashi and Takahagi 2015). Thus, the results of XPS made it possible to evaluate the mutual share of $\mathrm{sp}^{2}$ and $\mathrm{sp}^{3}$ hybridized carbon. (Lopez-Santos et al. 2009; Tashiro et al. 2013; Kaczorowski et al. 2015). The quantitative analysis results of deconvoluted spectra are presented in Table 3.

Table 2 - The content of oxygen, carbon and nitrogen on the PU surface in both cases: before and after the modification.

\begin{tabular}{cccc}
\multicolumn{4}{c}{ The percentage contents of chemical elements [\% at] } \\
& MW/RF & MS/RF & PU \\
\hline O1s & 10.10 & 16.40 & 21.50 \\
C1s & 89.03 & 82.14 & 77.59 \\
N1s & 0.87 & 1.46 & 0.89 \\
\hline
\end{tabular}

Table 3 - The quantitative analysis of the deconvolution of C1s peaks of carbon-based coatings synthesized using MW/RF and RF/MS techniques.

\begin{tabular}{ccccc}
\hline \multicolumn{3}{c}{ The analysis of deconvolution of C1s peak } \\
\cline { 2 - 5 } & \multicolumn{3}{c}{ MW/RF } & \multicolumn{2}{c}{ RF/MS } \\
\cline { 2 - 5 } & {$[\%]$} & $\begin{array}{c}\text { FWHM } \\
{[\mathrm{eV}]}\end{array}$ & {$[\%]$} & $\begin{array}{c}\text { FWHM } \\
{[\mathrm{eV}]}\end{array}$ \\
\hline $\mathrm{sp}^{2}(284.5 \mathrm{eV})$ & 57.77 & 1.62 & 56.63 & 1.64 \\
$\mathrm{sp}^{3}(285.3 \mathrm{eV})$ & 32.16 & 3.01 & 27.19 & 3.77 \\
$\mathrm{C}-\mathrm{O}(286.1 \mathrm{eV})$ & 7.86 & 2.30 & 8.40 & 2.18 \\
$\mathrm{C}=\mathrm{O}(288 \mathrm{eV})$ & 2.20 & 2.90 & 7.78 & 3.67 \\
$\mathrm{sp}^{3}\left(\mathrm{sp}^{2}+\mathrm{sp}^{3}\right)$ & 0.36 & - & 0.32 & - \\
\hline
\end{tabular}



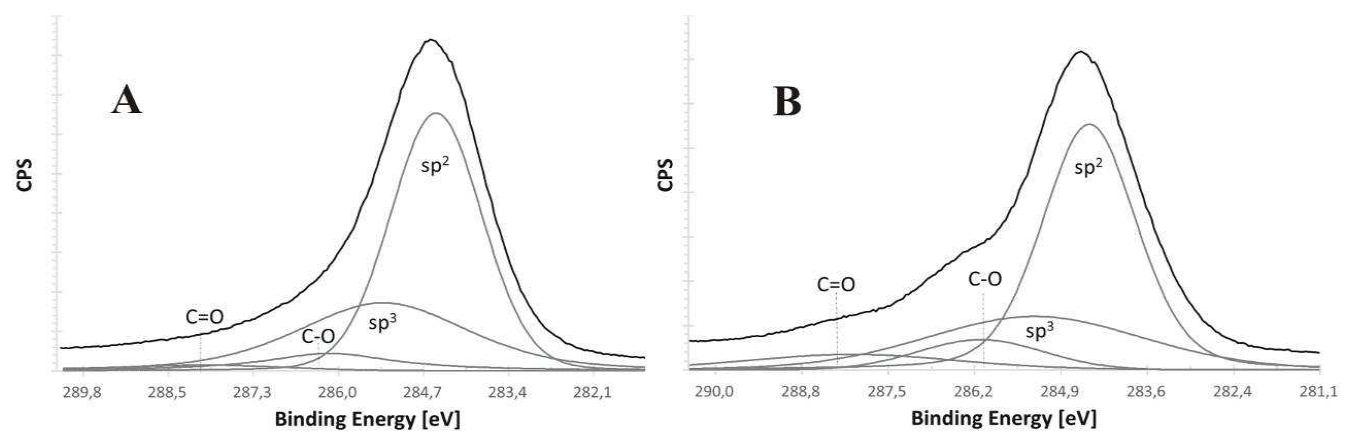

Figure 3 - The deconvolution of XPS C1s peaks of: a) substrate modified with use of MW/RF method, b) substrate modified with use of RF/MS method.

Figure 4 presents typical Raman spectra of manufactured carbon coatings with their deconvolution employing four-peak fitting method. Registered Raman spectra were deconvoluted using four peak fitting method and four Gaussians, attributed to D1 $\left(1180 \mathrm{~cm}^{-1}\right.$ characteristic for nanocrystalline diamond), D2 $\left(1350 \mathrm{~cm}^{-1}\right.$ characteristic for microcrystalline or nanocrystallinegraphite), G1 (1530 - $1571 \mathrm{~cm}^{-1}$ characteristic for disordered graphite) and G2 (1590 $\mathrm{cm}^{-1}$ characteristic for ordered graphite) (Tai et al. 2009).

\section{Contact Angle and Surface Free Energy Analysis}

Table 4 shows the contact angles of polar and nonpolar liquid drops together with calculated values of polar and dispersive component of the total surface free energy for the analyzed samples. The values did not show any significant changes in surface hydrophobicity. Both the substrate and coatings were characterized by very high water contact angles. The difference in the surface properties between the two types of modification was visible in their interaction with non-polar liquid drop. Contact angle for diiodomethane for PU sample was $\sim 23^{\circ}$. Further modification of polyurethane substrate resulted in increased value of contact angle up to $27.51^{\circ}$ for MW/RF sample and up to $68.5^{\circ}$ for RF/MS sample. Such variety of interactions between the samples and polar and non-polar liquids was reflected in the values of the total surface free energy.The total SFE for polyurethane substrate was equal to $48.67 \mathrm{~mJ} / \mathrm{m}^{2}$, whereas for samples modified with MW/RF and RF/MS techniques its value decreased to 45.71 $\mathrm{mJ} / \mathrm{m}^{2}$ and $24.15 \mathrm{~mJ} / \mathrm{m}^{2}$, respectively.
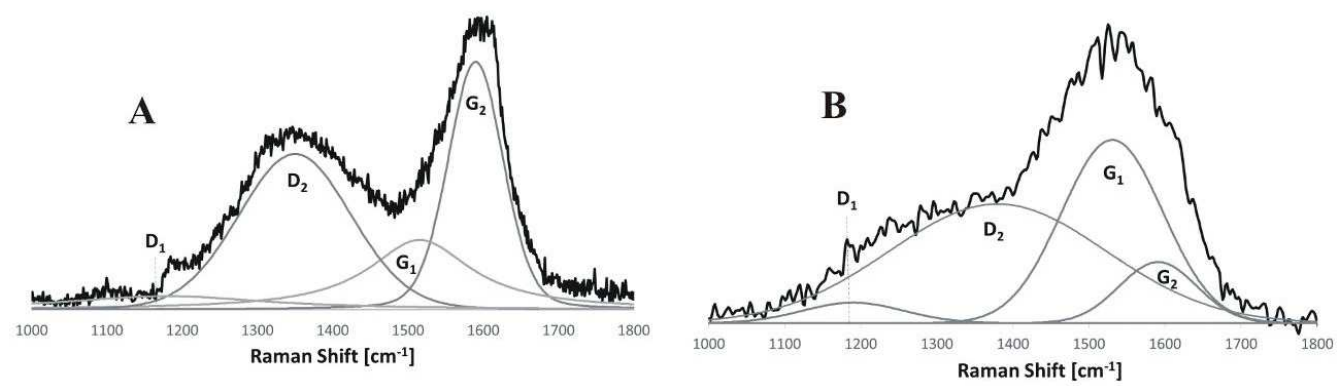

Figure 4 - Raman spectra of examined samples for a) MW/RF method and b) MS/RF method.

Table 4 - Contact angles of polar and non-polar liquid drops and polar and dispersive component of the total surface free energy for the analyzed samples.

\begin{tabular}{ccccc}
\hline \multirow{2}{*}{ Sample } & $\begin{array}{c}\text { Water } \\
\text { Avg. Contact angle }\end{array}$ & $\begin{array}{c}\text { Diiodomethane } \\
\text { Avg. Contact angle }\end{array}$ & $\begin{array}{c}\text { Surface free energy } \\
\text { Polar component }\end{array}$ & Dispersive component \\
\hline PU & $104.90 \pm 2.80$ & $22.90 \pm 1.50$ & 0.56 & 48.11 \\
MW/RF & $105.93 \pm 1.53$ & $27.51 \pm 2.03$ & 0.49 & 45.22 \\
RF /MS & $104.25 \pm 0.85$ & $68.50 \pm 6.14$ & 0.43 & 23.72 \\
\hline
\end{tabular}




\section{Biological Evaluation}

All the parameters summarizing biological evaluation of the studied surfaces are shown in Table 5. Experimental whole blood shear stress resulted in a decrease in the number of platelets, which was the highest for MW/RF coating (by $47.3 \%$ ) but similar for polyurethane and RF/MS surface (37.5 and 38.3\%, respectively). Both $\mathrm{MW} / \mathrm{RF}$ and polyurethane induced aggregation of the platelets, as evidenced by flow cytometry. Aggregation of the blood platelets corresponds to initiation of the first step of thrombotic event and is regarded as a predictor for major adverse cardiovascular events. The smallest fraction of aggregates was noticed for RF/MS surface $(1.8 \pm 1.5$ vs. $7.4 \pm 8.74$ for polyurethane and $5.3 \pm 10.37$ for $\mathrm{MW} / \mathrm{RF}$ ) and this difference was the highest for the aggregates composed of more than two platelets. Fraction of activated platelets, as measured by the expression of P-selectin, was the lowest for RF/MS coating (7.9\% \pm 6.99$)$, while the reference polyurethane and MW/RF surfaces $(12.4 \% \pm 14.53$ and $13.0 \% \pm 12.25)$ resulted in a similar percentage of activated platelets. Both the tested carbon nanocoatings generated lower number of plateled microparticles during whole blood shear stress

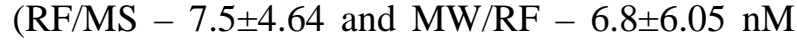
phosphatydylserine $/ \mathrm{mL})$, than the reference polyurethane (10.1 \pm 5.94$)$.

Another very important parameter characterizing the surface biocompatibility is susceptibility for microbial colonization. The surface of PU was more susceptible for $E$. coli colonization than the modified surfaces. The number of bacterial cells found on the PU surface, in the field of view, was four times higher than on the modified surfaces. In addition, surface area occupied by $E$. coli cells on PU sample (48.7\%) was larger than for RF/MS (18.8\%) and for MW/RF (26.1\%). Any material intended to cooperate with the blood circulatory system should be well tolerated by the endothelial cells. In this sense, PU poorly met this requirement. The cell viability of endothelial cells was relatively low $(71.43 \%)$. In contrast, the modified surfaces allowed for much better cell viability reaching 96.17 and $97.14 \%$, respectively.

Table 5 - Parameters summarizing biological evaluation of the studied surfaces.

\begin{tabular}{lccc}
\multicolumn{1}{c}{ Parameter } & PU & RF/MS & MW/RF \\
\hline $\begin{array}{l}\text { Decrease in the platelet number caused by shear stress (\% of } \\
\text { initial) }\end{array}$ & 37.5 & 38.3 & 47.3 \\
$\begin{array}{l}\text { Fractionofplateletaggregates (\%) } \\
\text { Fraction of activated platelets (\%) }\end{array}$ & $7.4 \pm 8.74$ & $1.8 \pm 1.5$ & $5.3 \pm 10.37$ \\
$\begin{array}{l}\text { Fraction of platelet derived microparticles } \\
\text { (nMphosphatydylserine/mL) }\end{array}$ & $12.4 \pm 14.53$ & $7.9 \pm 6.99$ & $13.0 \pm 12.25$ \\
$\begin{array}{l}\text { Bacterial colonization number of } \text { E. coli cells in the field of } \\
\text { view (\% of control) }\end{array}$ & $10.1 \pm 5.94$ & $7.5 \pm 4.64$ & $6.8 \pm 6.05$ \\
$\begin{array}{l}\text { Bacterial colonization surface occupied by E. coli cells (\%) } \\
\text { Viability of endothelial cells (\%) }\end{array}$ & $48.7 \pm 4.6$ & $18.8 \pm 2.3$ & $26.1 \pm 2.1$ \\
\hline
\end{tabular}

\section{DISCUSSION}

Presented results are the part of ongoing research on the modification of the surface of polyurethane. Samples used in the present study were selected in terms of the best mechanical properties and particularly resistance to wear, which already has been published earlier (Kaczorowski et al. 2009). The structures of carbon coatings were clearly visible in the TEM examination results. The coating produced using MW/RF technique was amorphous, whereas in case of the coating synthesized by RF/MS method, its gradient character, including nano-composite $\mathrm{Ti}^{-} \mathrm{Ti}_{\mathrm{x}} \mathrm{C}_{\mathrm{y}}-\mathrm{DLC}$ interlayer was visible. Application of the chemical composition gradient together with the nano-composite structure of the middle interlayer possibly decreased the stress and by facilitating the interfacial sliding between the neighbouring grain-matrices subsequently increased the ductility of the film (Wang et al. 2009) and probably ensured a better adhesion to soft polymer substrate. The present results of the AFM research proved that there was a big influence of conducted modifications on the surface development of polyurethane substrates. This, in turn, should be considered in accordance to two different phenomena taking place during the modification process. Namely etching and synthesis processes. These processes take place simultaneously and their influence is noticeable in 
case of all types of carbon coatings. The changes in the roughness parameters depend on the number of modification stages. In case of one stage - MW/RF modifications, they are lower than for three stage $\mathrm{RF} / \mathrm{MS}$ techniques. It is mainly influenced by the preliminary etching procedure, performed in oxygen/argon plasma. In this case, the surface development is higher and the surface topography is more varied. It is mainly connected with the chemical character of the interaction between oxygen free-radicals and modified substrate. In this field, oxygen plasma is known to be one of the most aggressive agents in polymer degradation, or modification (Vesel et al. 2014). Resulting surface chemical structure may possibly improve the adhesion of the coating by increasing free surface energy of PU substrate by bonding oxygen to carbon atoms on their surface (Hauert 2004). The topography of carbon coatings depends on their thickness and is related to the residual stress (Nakahigashi et al. 2004). In the present case, the gradient character of $\mathrm{Ti}-\mathrm{Ti}_{\mathrm{x}} \mathrm{C}_{\mathrm{y}}$ interlayer might also affect this parameter. Analysing the chemical composition of proposed layers, it would be worth to emphasize that in case of both MW/RF and RF/MS modifications in the manufactured layer, there was lack of other elements characteristic for the substrate and the interlayer as well. The only contamination was nitrogen adsorbed on the surface form air. The XPS studies showed different $\mathrm{sp}^{3} /\left(\mathrm{sp}^{2}+\mathrm{sp}^{3}\right)$ ratio for both the modification techniques, wherein higher $\mathrm{sp}^{3}$ coordinated carbon concentration was registered for the sample MW/RF. This fact, together with the lower self-bias applied during the deposition process, proves the $\mathrm{sp}^{3}$ promoting properties of the microwave plasma (Kaczorowski and Niedzielski 2008). The $\mathrm{sp}^{3}$ fraction and hydrogen content are well known to be the factors that determine the mechanical properties of carbon coatings; however, high $\mathrm{sp}^{3}$ content may also affect the adhesion, causing the coating delamination (Lackner et al. 2009; Robertson 2002). Higher concentration of $\mathrm{sp}^{2}$ bonded carbon (graphite-like) obviously decreases the mechanical properties; however, ensures the good flexibility of the coating, which may be critical in this application (Ma et al. 2007). C1 score level of deconvolution results did not significantly differ in terms of contribution and binding energy of particular peaks; however, higher intensity and FWHM of peaks denoted as $\mathrm{C}-\mathrm{O}$ and $\mathrm{C}=\mathrm{O}$ for the sample $\mathrm{RF} / \mathrm{MS}$ clearly indicated the increased concentration of these functional groups (Matsumoto et al. 2008).
Based on results from the Raman spectroscopy and deconvolution, the ID/IG ratio was determined, wherein $\mathrm{D}=\mathrm{D} 1+\mathrm{D} 2$ and $\mathrm{G}=\mathrm{G} 1+\mathrm{G} 2$. For the coatings manufactured by means of $\mathrm{MW} / \mathrm{RF}$ plasma, the ID/IG was equal to 0.69 , whereas for hybrid MF/MS coatings, it was 0.84 . This increase indicated the lowering trend in concentration of $\mathrm{sp}^{3}$ hybridized carbon and was in agreement with the XPS results. Water contact angle for the analysed samples did not change significantly. Samples before and after the modification remained strongly hydrophobic; however, noticeable change in the surface free energy was observed. There was lowering trend in the polar and dispersive component of SFE. The lowest value of the dispersive component (observed for the sample RF/MS) was more than two times lower than that of polyurethane. A low blood-biomaterial interfacial tension is beneficial to blood compatibility (Kwok et al. 2005). Very low value of polar component of the surface free energy was also expected to enhance the hemocompatibility (Kwok et al. 2007). Biological evaluation of the surface-modified polyurethane provides a number of important information. Though presented differences were not statically significant due to a great variability in the platelet response to the shear stress, RF/MS coating was superior in hemocompatibility properties over the reference polyurethane surface. During the dynamic tests of platelets activation, consistently lower platelet consumption, selectin-P activation and platelet microparticles generation was observed for this coating. Long-term adverse clinical effects of the implantable devices in contact with blood are consequences of the thrombotic events. Even moderate improvements in hemocompatibility parameters, as evidenced for the RF/MS carbon coating by the lowest number of platelet aggregates and decreased potential for platelet microparticles generation, could reduce the cumulative risk of the stroke, or other arterial occlusion. Although the current results did not reach the statistical significance, this could be explained by a low number of tested samples $(n=6)$, and a high dispersion of results obtained for the reference polyurethane and MW/RF samples. The PU surface is much more susceptible for microbial colonization than the modified surfaces. It is extremely important, when an implanted medical device is predicted to be used for a long time. A high susceptibility to colonization by the opportunistic microbes present in the patient's body can result in recurrent opportunistic infections, and 
finally require re-implantation. From the medical safety aspects, both the modified surfaces showed much better properties than the unmodified polyurethane. Considering the contact and integration of medical device with the blood circulating system, attention should be paid on the viability of endothelial cells in contact with the artificial surface. In such a case, the unmodified polyurethane surface strongly reduces the viability of endothelial cells, which may cause necrosis in the place of contact of the vessel wall with the artificial surface. Both the modified surfaces exhibited much better properties in contact with the endothelial cells, thus were more suitable for a direct contact with endothelium. Both modified surfaces showed similar differences in relation to the unmodified polyurethane, but also between the carbon-coated surfaces, there were visible evident differences. RF/MS coating surface seemed to be better situated as a potential polyurethane competitor. It caused a lower platelet activation and aggregation being more resistant to the microbial colonization. The MW/RF coating surface was slightly more suitable for the contact with endothelium. It could be also worth noting that the technologies presented here provided the ability to produce a surface with the desired properties.

\section{CONCLUSIONS}

Results showed that both the modification methods influenced the surface development. The interface between the structures of synthesized coatings and modified surfaces presented the continuous character. Results of XPS indicated that the synthesized coatings consisted of c.a. 32-36\% of sp ${ }^{3}$ hybridized carbon. The dynamic tests showed the superiority of modification over the starting material. The applied carbon coatings reduced the bacterial colonization and improved the endothelial cell viability. The character of changes of the physicochemical and biological properties of the proposed coatings was similar, regardless their structure. Despite the discrepancies in the results obtained by the different biological tests, achieved outcomes proved that polyurethane modified with the use of carbon layers could constitute an interesting solution for biomedical application.

\section{ACKNOWLEDGMENT}

This work was financially supported by the MultiYear Program for years 2007-2011 "Polish ArtificialHeart" and "Development of the type series of single disk artificial heart valves for paediatric ventricular assist devices" research project (NR13-0118-10/2011) by The National Centre for Research and Development.

\section{REFERENCES}

Asakawa R, Nagashima S, Nakamura Y, Hasebe T, Suzuki T, Hotta A. Combining polymers with diamond-like carbon (DLC) for highly functionalized materials. Surf Coat Tech. 2011; 206:676-685.

Batory D, Blaszczyk T, Clapa M, Mitura S. Investigation of anti-corrosion properties of $\mathrm{Ti}$ : $\mathrm{C}$ gradient layers manufactured in hybrid deposition system. J Mater Sci. 2008; 43:3385-3391.

Batory D, Gawronski J, Kaczorowski W, Niedzielska A. C-HAp composite layers deposited onto AISI 316L austenitic steel. Surf Coat Tech. 2012; 206:2110-2114.

Batory D, Szymanski W, Clapa M. Mechanical and tribological properties of gradient a-C:H/Ti coatings. Mater Sci-Poland. 2013; 31:415-423.

Batory D, Gorzedowski J, Rajchel B, Szymanski W, Kolodziejczyk L. Silver implanted diamond-like carbon coatings. Vacuum.2014; 110:78-86.

Batory D, Jedrzejczak A, Kaczorowski W, Szymanski W, Kolodziejczyk L, Clapa M, et al. Influence of the process parameters on the characteristics of siliconincorporated a-C:H:SiOx coatings. Surf Coat Tech. 2015; 271:112-118.

Bazaka K, Jacob MV, Crawford RJ, Ivanova EP. Plasma-assisted surface modification of organic biopolymers to prevent bacterial attachment. Acta Biomater. 2011; 7:2015-2028.

Bozukova D, Pagnoulle C, Jerome R, Jerome C. Polymers in modern ophthalmic implants-Historical background and recent advances. Mater Sci Eng $R$. 2010; 69:63-83.

Courtney JM, Lamba NMK, Sundaram S, Forbes CD. Biomaterials for blood-contacting applications. Biomaterials. 1994; 15:737-744.

Desmet T, Morent R, De Geyter N, Leys C, Schacht E, Dubruel P. Nonthermal plasma technology as a versatile strategy for polymeric biomaterials surface modification: a review. Biomacromolecules.2009; 10:2351-2378.

Fedel M, Motta A, Maniglio D, Migliaresi C. Surface properties and blood compatibility of commercially available diamond-like carbon coatings for cardiovascular devices. J Biomed Mater Res B. 2009; 90B:338-349.

Foong YM, Koh ATT, Ng HY, Chua DHC. Mechanism behind the surface evolution and microstructure changes of laser fabricated nanostructured carbon 
composite. J Appl Phys. 2011; 110:054904-1- 0549048.

Hauert R. An overview on the tribological behavior of diamond-like carbon in technical and medical applications. Trib Int. 2004; 37:991-1003.

Hauert R, Muller U. An overview on tailored tribological and biological behavior of diamond-like carbon. Diam Relat Mater. 2003; 12:171-177.

Jakubowski W, Bartosz G, Niedzielski P, Szymanski W, Walkowiak B. Nanocrystalline diamond surface is resistant to bacterial colonization. Diam Relat Mater. 2004; 13:1761-1763.

Jones DS, Garvin CP, Dowling D, Donnelly K, Gorman SP. Examination of surface properties and in vitro biological performance of amorphous diamond-like carbon-coated polyurethane. J Biomed Mater Res. B. 2006; 78B:230-236.

Junkar I, Vesel A, Cvelbar U, Mozetic M, Strnad S. Influence of oxygen and nitrogen plasma treatment on polyethylene terephthalate (PET) polymers. Vacuum. 2009; 84:83-85.

Kaczorowski W, Niedzielski P. Morphology and growth process of carbon films prepared by microwave/radio frequency plasma assisted CVD. Adv Eng Mater. 2008; 10:651-656.

Kaczorowski W, Batory D, Niedzielski P. Application of microwave/radio frequency and radio frequency/magnetron sputtering techniques in polyurethane surface modification. JAMME. 2009; 37: 286-291.

Kaczorowski W, Szymanski W, Batory D, Niedzielski P. Tribological properties and characterization of diamond like carbon coatings deposited by MW/RF and RF plasma-enhanced cvd method on poly(etherether-ketone). Plasma Processes Polym. 2014; 11:878-887.

Kaczorowski W, Szymanski W, Batory D, Niedzielski P. Effect of plasma treatment on the surface properties of polydimethylsiloxane. J App Pol Sci. 2015; 132:2, 41635, doi: 10.1002/app.41635

Kleinen L, Boede U, Laube N. Ex-vivo investigations on the friction behavior of amorphous carbon coated ureteral stents. Diam Relat Mater. 2008; 17:17461750.

Kwok SCH, Jin W, Chu PK. Surface energy, wettability, and blood compatibility phosphorus doped diamondlike carbon films. Diam Relat Mater. 2005; 14:78-85.

Kwok SCH, Zhang W, Wan GJ, McKenzie DR, Bilek MMM, Chu PK. Hemocompatibility and anti-bacterial properties of silver doped diamond-like carbon prepared by pulsed filtered cathodic vacuum arc deposition. Diam Relat Mater. 2007; 16:1353-1360.

Lackner JM, Major R, Major L, Schoeberl T, Waldhauser W. RF deposition of soft hydrogenated amorphous carbon coatings for adhesive interfaces on highly elastic polymer materials. Surf Coat Tech. 2009; 203:2243-2248.
Lackner JM, Waldhauser W, Hartmann P, Bruckert F, Weidenhaupt M, Major R, et al. Hemocompatibility of inorganic physical vapor deposition (PVD) coatings on thermoplastic polyurethane polymers. $J$ Funct Biomater. 2012; 3:283-297.

Laube N, Kleinen L, Bradenahl J, Meissner A. Diamondlike carbon coatings on ureteral stents - A new strategy for decreasing the formation of crystalline bacterial biofilms? J Urology. 2007; 177:1923-1927.

Lopez-Santos C, Yubero F, Cotrino J, Contreras L, Barranco A, Gonzalez-Elipe AR. Formation of nitrogen functional groups on plasma treated DLC. Plasma Processes Polym. 2009; 6:555-565.

Ma WJ, Ruys AJ, Mason RS, Martin PJ, Bendavid A, Liu Z, et al. DLC coatings: Effects of physical and chemical properties on biological response. Biomaterials 2007; 28:1620-1628.

Martinez-Martinez D, De Hosson J.Th.M. On the deposition and properties of DLC protective coatings on elastomers: A critical review. Surf Coat Tech. 2014; 258:677-690.

Matsumoto R, Sato K, Ozeki K, Hirakuri K, Fukui Y. Cytotoxicity and tribological property of DLC films deposited on polymeric materials. Diam Relat Mater. 2008; 17:1680-1684.

Mitura K, Niedzielski P, Bartosz G, Moll J, Walkowiak B, Pawlowska Z, et al. Interactions between carbon coatings and tissue. Surf Coat Tech. 2006; 201:21172123.

Myllymaa K, Levon J, Tiainen V-M, Myllymaa S, Soininen A, Korhonen $\mathrm{H}$, et al. Formation and retention of staphylococcal biofilms on DLC and its hybrids compared to metals used as biomaterials. Colloid Surface B. 2013; 101:290-297.

Nakahigashi T, Tanaka Y, Miyake K, Oohara H. Properties of flexible DLC film deposited by amplitude-modulated RF P-CVD. Trib Int. 2004; 37:907-912.

Narayan RJ. Nanostructured diamondlike carbon thin films for medical applications. Mat Sci Eng C. 2005; 25:405-416.

Ohgoe Y, Hirakuri KK, Tsuchimoto K, Friedbacher G, Miyashita O. Uniform deposition of diamond-like carbon films on polymeric materials for biomedical applications. Surf Coat Tech. 2004; 184:263-269.

Robertson J. Diamond-like amorphous carbon. Mat Sci Eng R. 2002; 37:129-281.

Rudawska A, Jacniacka E. Analysis for determining surface free energy uncertainty by the Owen-Wendt method. Int J Adhes Adhes. 2009; 29:451-457.

Sanak M, Jakiela B, Wegrzyn W. Assessment of hemocompatibility of materials with arterial blood flow by platelet functional tests. Bull Polish Acad Sci Tech Sci. 2010; 58:317-322.

Schroeder A, Francz G, Bruinink A, Hauert R, Mayer J, Wintermantel E. Titanium containing amorphous hydrogenated carbon films (a-C : H/Ti): surface analysis and evaluation of cellular reactions using 
bone marrow cell cultures in vitro. Biomaterials. 2000; 21:449-456.

Tai FC, Lee SC, Chen J, Wei C, Chang SH. Multipeak fitting analysis of Raman spectra on DLCH film. $J$ Raman Spectrosc. 2009; 40:1055-1059.

Takabayashi S, Takahagi T. Surface oxidation process of a diamond-like carbon film analyzed by difference Xray photoelectron spectroscopy. Surf Interf Anal. 2015; 47:345-349.

Tashiro H, Nakaya M, Hotta A. Enhancement of the gas barrier property of polymers by DLC coating with organosilane interlayer. Diam Relat Mater. 2013; 35:7-13.

Vesel A, Kolar M, Stana-Kleinschek K, Mozetic M. Etching rates of blood proteins, blood plasma and polymer in oxygen afterglow of microwave plasma. Surf Interface Anal. 2014; 46:1115-1118.

Wang C, Yu X, Hua M. Microstructure and mechanical properties of Ag-containing diamond-like carbon films in mid-frequency dual-magnetron sputtering. App Surf Sci. 2009; 256:1431-1435.

Received: June 10, 2015; Accepted: September 07, 2015. 